\title{
A frequency method for dynamic uncertainty evaluation of measurement during modes of dynamic operation
}

\author{
O.M. Vasilevskyi*
}

Department of Metrology and industrial automation, Vinnytsia National Technical University, 95 Khmelnitskoye Shose, Vinnytsia, 21021 Vinnytsia, Ukraine

Received: 2 March 2015 / Accepted: 30 May 2015

\begin{abstract}
Proposed the frequency of method to assessing the dynamic uncertainty of measurement devices which allows to investigate the accuracy of changes in the dynamic operation conditions in the frequency domain, estimate the amplitude dynamic uncertainty based on frequency characteristic and the spectral function of the input signal. This approach was tested at the evaluation dynamic uncertainty of devices measuring dynamic torque of induction electromotors, whereby it was confirmed correctness theoretical statements. On the basis of the calculations that the relative dynamic uncertainty of device measuring is $5.38 \%$ and the measuring range from 0.1 to $8 \mathrm{~N} \mathrm{~m}$, and in the range measuring of dynamic torque of electromotors from 0.1 to $30 \mathrm{~N} \mathrm{~m}$ its value is $5.33 \%$. The proposed approach to the evaluation of the dynamic uncertainty can be used for measuring instruments that have dynamic elements of any type. First proposed a theoretical approach to the assessment the characteristics dynamic of the uncertainty of measuring instruments, working in dynamic modes of operation that meets international standards for presentation of the characteristics of quality and accuracy of electrical products. Theoretical results are tested when measuring dynamic torque of electromotors, which confirmed the correctness and effectiveness of the proposed frequency method for estimating dynamic uncertainty.
\end{abstract}

Keywords: Dynamic uncertainty of measurement devices, assessment of uncertainties in dynamic mode of operation, quality assurance of dynamic measurements, spectral function, frequency characteristic

\section{Introduction}

In producing reports on the results of dynamic measurement we must state the quantitative value of each experimental quality so that its reliability can be correctly assessed [1-5]. Without this value, the results obtained of dynamic measurement cannot be compared, and neither with analogous results obtained by leading laboratories around the world. Therefore, we will present a method for the evaluation of dynamic uncertainty of measurement means to provide the results of dynamic measurement and their comparison. This method will enable the uncertainty of measurement means to be calculated when working in a dynamic mode.

In the current and generally accepted concept of the uncertainty of the results of measurement, it may be shown how to process and express the results of static measurements, however the methods of expressing dynamic uncertainty of measurement means based on the concept of uncertainty has not been given sufficient attention. It is known that here are studies [6-8], which propose

^ Correspondence: o.vasilevskyi@gmail.com the use of the classical theory of determining dynamic error for the calculation of dynamic uncertainty. Therefore, the development of a new method for evaluation the dynamic uncertainty that is introduced by the measurement means during its operation in a dynamic mode, becomes a key scientific task, the resolution of which may ensure the uniformity of measurements in this field.

Given the above, the purpose of this paper is to describe the development of a new method for evaluating the dynamic uncertainty of measurement means, which can be used within the concept of uncertainty to assess the precision of measurement means operating in a dynamic mode.

During the process of measurement of physical quantities there is always a transition mode in the operation of measuring instruments, during which the signal at the output changes significantly over time $[9,10]$. This fact can be explained by the inertial properties of the measurement means, as they generally are incorporated in a set of different masses and springs, capacities and inductances or other inert elements that cause the appearance of uncertainty in dynamic modes [10]. 
In addition, signal delay may be observed in a digital measurement device, which is caused by the completion time of its transformation into a digital form. This leads to the unacceptability of the equation for the transformation of measurement means that reflects its static nature. In this case, we need to use differential equations that describe the dynamic relationship of the output $y(t)$ and the input $x(t)$ values of the measurement means. In deriving the differential equations, the input signals are incorporated in the right side, as they are themselves the reason for the operation to take place, and the left side of the differential equation contains data from the output signal (measurement means response) $[10,11]$

$$
\sum_{i=0}^{n} a_{i} y^{i}(t)=\sum_{k=0}^{m} b_{k} x^{k}(t),
$$

where $x(t), y(t)$ are respectively, the input and output values; $i, k$ the order of the derivatives; $a, b$ the coefficients characterizing the properties of the measurement means.

To represent the differential equation in the frequency domain, the symbol for the differentiation of coordinate time $\frac{d}{d t}$ is replaced by $j \omega$, and equation (1) takes the form

$$
\frac{y(j \omega)}{x(j \omega)}=S_{0} \frac{b_{m}(j \omega)^{m}+b_{m-1}(j \omega)^{m-1}+\ldots+1}{a_{n}(j \omega)^{n}+a_{n-1}(j \omega)^{n-1}+\ldots+1},
$$

where $y(j \omega), x(j \omega)$ are respectively, the spectral functions of the input and output of the measured signal; $S_{0}=b_{0} / a_{0}$ is the static sensitivity, i.e. the sensitivity of the constant input values (where $j \omega=0$ ); $S(j \omega)$ is the transfer function of the measurement means or operation sensitivity.

The most typical characteristics for measurement means are dynamic characteristics, described by differential equations of the first and second order, and in some cases, third and higher orders $[10,11]$.

Information on dynamic characteristics needs to be included in the documentation on the measurement means, although if such information is not available, it can be obtained on the basis of a priori information about the measurement means.

It is known that a measuring device with no inertia whatsoever, an ideal measurement transducer, has a frequency response in the form of [11]

$$
S(j \omega)=K,
$$

where $K$ is the transfer coefficient of the measurement means.

Measurement devices which have aperiodic components (measurement transducers of temperature into resistance) may be described by a frequency characteristic in the form of [11]

$$
S(j \omega)=\frac{K}{1+j \omega \tau},
$$

where $\tau$ is the time constant, determined by the parameters of the measurement means.

Measurement equipment with an integration component (integrator amplifiers) may be described by the frequency characteristic form [11]

$$
S(j \omega)=\frac{K}{j \omega} .
$$

Measurement means with a boosting component (differential amplifiers) may be described by the frequency characteristic form [11]

$$
S(j \omega)=K(1+j \omega \tau) .
$$

Measurement means with a delay component (analogueto-digital converters) may be described by the frequency characteristic form [11]

$$
S(j \omega)=\exp (-j \omega \tau) .
$$

Measurement means with an oscillating component (electromechanical measurement transducers) may be described by the frequency characteristic form [11]

$$
S(j \omega)=\frac{K}{1+j \omega \tau_{1}-\omega^{2} \tau_{2}^{2}} .
$$

Taking into account the known frequency characteristics of the measurement means, we will develop a method for the evaluation of the dynamic uncertainty of measurement means as described below.

\section{An approach to the evaluation of the dynamic uncertainty of measurement means}

Frequency characteristics provide a practical means for the evaluation of dynamic uncertainty of measurement means in a readily accessible manner.

As a rule, information on the dynamic characteristics of the measurement means can be located in the documentation accompanying such devices, although if such information is not available, it can obtained from the metrological certification of the measurement means or it may be determined a priori $[5,11]$.

Dynamic measurement uncertainty is defined as a factor of measurement uncertainty, which is caused by a response by the measurement means to the frequency (speed) of the measurement of the input variable (input signal), which itself depends on the dynamic properties of the measurement means and the frequency spectrum of the input signal $[11,12]$.

The dynamic uncertainty, $u_{\mathrm{D}}$, is estimated by the formula

$$
u_{D}=\sqrt{\frac{1}{2 \pi} \int_{-\infty}^{\infty}|S(j \omega)|^{2}|X(j \omega)|^{2} d \omega}
$$


where $|S(j \omega)|$ is the modulus of the frequency response of the Measurement Means Device (MMD), which is used for dynamic measurements, or the frequency amplitude characteristics of the measurement means device (MMD), which may be determined by the formula

$$
|S(j \omega)|=\sqrt{a^{2}(\omega)+b^{2}(\omega)},
$$

where:

$a(\omega), b(\omega)$ are respectively the real and imaginary parts of the frequency characteristics $S(j \omega)$;

$X(j \omega)$ is the spectrum function of the input signal associated with the input time function $x(t)$ in the Laplace expression:

$$
X(j \omega)=\int_{0}^{\infty} x(t) e^{-j \omega_{0} t} d t
$$

where $\omega_{0}$ is the frequency of the input signal.

The upper limit of the integral equation (11) for a finite time interval can be changed for the total observation time $T$.

If the measured signal $x(t)$ is discrete, then in equation (11), the integration sign can be replaced by a summation sign, having performed the following substitutions: $t$ is replaced by $n T_{\mathrm{a}}$, where $n$ varies from 0 to $N-1$; with $T_{\mathrm{a}}$ denoting the sampling period, when $x(t)$ then takes the form $w\left(n T_{\mathrm{a}}\right)$, and $e^{-j \omega_{0} t}$ is replaced by $e^{-j \omega_{0} n T_{\mathrm{a}}}$.

After such replacements, equation (11) can be presented in the following discrete form:

$$
\begin{aligned}
X_{d}(j \omega) & =\sum_{n=0}^{N-1} x\left(n T_{\mathrm{a}}\right) e^{-j \omega_{0} n T_{\mathrm{a}}} \\
& =\sum_{n=0}^{N-1} x\left(n T_{\mathrm{a}}\right) \cos \omega_{0} n T_{\mathrm{a}}-j \sum_{n=0}^{N-1} x\left(n T_{\mathrm{a}}\right) \sin \omega_{0} n T_{\mathrm{a}}
\end{aligned}
$$

where,

$$
\omega_{0}=\frac{2 \pi}{N T_{\mathrm{a}}} K, a K=0,1, \ldots, N-1 .
$$

In order that the discrete spectral function corresponds by its value to the continuous spectral function, it is necessary to multiply it by the sampling interval represented by:

$$
X(j \omega)=T_{\mathrm{a}} X_{d}(j \omega) .
$$

For dynamic measurement of discrete time signals, the equation for the calculation of the dynamic uncertainty (9), taking into account equations (12) and (13) may be written as:

$$
u_{D}=\sqrt{\frac{T_{\mathrm{a}}}{N} \sum_{\mathrm{k}=0}^{N-1} \sum_{n=0}^{N-1} x^{2}\left(n T_{\mathrm{a}}\right) e^{-j \frac{4 \pi n k}{N}} A^{2}\left(k \frac{2 \pi}{N T_{\mathrm{a}}}\right)},
$$

where:

$$
A\left(k \frac{2 \pi}{N T_{\mathrm{a}}}\right)=A(\omega)=|S(j \omega)|
$$

is the amplitude frequency characteristic of the measurement means;

$\Delta \omega=\frac{2 \pi}{N T_{\mathrm{a}}}$ is the difference between the discrete values of the frequencies;

$T_{\mathrm{a}}$ is the time sampling;

$N$ is the number of samples;

$N T_{\mathrm{a}}$ is the total observation time.

So, to express dynamic uncertainty it is necessary to determine the frequency response modulus of the measurement means used for the measurements, and the spectral function of the input signal, which is associated with the measurement signal in the time domain $x(t)$ of the Laplace transformation.

To confirm the above proposed theoretical statements, we will consider the evaluation of the dynamic uncertainty of the measurement means of the dynamic moment of an electric motor.

Literature sources $[9,12]$ provide us with the differential equation which describes the dynamic relationship of input and output signals during the functioning of the measurement means measuring the dynamic moment of an electric machine (EM):

$$
\frac{d^{2} \phi(t)}{d t^{2}}+2 v \omega_{p} \frac{d \phi(t)}{d t}+\omega_{p}^{2} \phi(t)=\frac{M_{c}(t)}{J_{c}},
$$

where:

$J_{C}$ is the total moment of inertia of the stator and the moving parts of the measuring transducer;

$v=\frac{P}{2 \sqrt{J_{c} C}}$ is the degree of calming of free oscillations of the measurement means;

$\omega_{p}=\sqrt{C / J_{c}}$ is the natural frequency (undamped) of the vibrations of the measuring transducer;

$C$ is the effort sensor stiffness;

$P$ is the calming coefficient.

The transfer function of the dynamic moment of the measurement means is described in reference [9]

$$
S(p)=\frac{K}{p^{2}+2 \nu \omega_{p} p+\omega_{p}^{2}}
$$

where:

$K=\frac{g K 1}{J_{c} \omega_{p}^{2}}$ is the coefficient of proportionality of the dynamic moment of the measurement means;

$g$ is gravity acceleration;

$K 1$ is the constant for the tensoresistive transducer.

Turning to the frequency domain and having separated the real and imaginary parts, as well as having conducted appropriate mathematical transformations, we may now 


$$
\begin{gathered}
|S(j \omega)|^{2}=\frac{K^{2}\left(\omega^{4}+4 \nu^{2} \omega^{2} \omega_{p}^{2}-2 \omega^{2} \omega_{p}^{2}+\omega_{p}^{4}\right)}{\omega^{8}+4 \omega^{6} \omega_{p}^{2}\left(2 \nu^{2}-1\right)+2 \omega^{4} \omega_{p}^{4}\left(3-8 \nu^{2}\right)+8 \nu^{2} \omega^{2} \omega_{p}^{4}\left(2 \nu^{2} \omega^{2}+\omega_{p}^{2}\right)-4 \omega^{2} \omega_{p}^{6}+\omega_{p}^{8}} \\
u_{D}=\left(\frac{1}{2 \pi} \int_{0}^{\infty} \frac{M_{k}^{2}\left(\omega^{2}+\nu^{2} \omega_{0}^{2}\right)}{\omega^{4}+2 \omega^{2} \nu^{2} \omega_{0}^{2}+\nu^{4} \omega_{0}^{4}} d \omega\right)^{\frac{1}{2}}\left(\int_{0}^{\infty} \frac{K^{2}\left(\omega^{4}+4 \nu^{2} \omega^{2} \omega_{p}^{2}-2 \omega^{2} \omega_{p}^{2}+\omega_{p}^{4}\right)}{\omega^{8}+4 \omega^{6} \omega_{p}^{2}\left(2 \nu^{2}-1\right)+2 \omega^{4} \omega_{p}^{4}\left(3-8 \nu^{2}\right)+8 \nu^{2} \omega^{2} \omega_{p}^{4}\left(2 \nu^{2} \omega^{2}+\omega_{p}^{2}\right)-4 \omega^{2} \omega_{p}^{6}+\omega_{p}^{8}} d \omega\right)^{\frac{1}{2}} .
\end{gathered}
$$

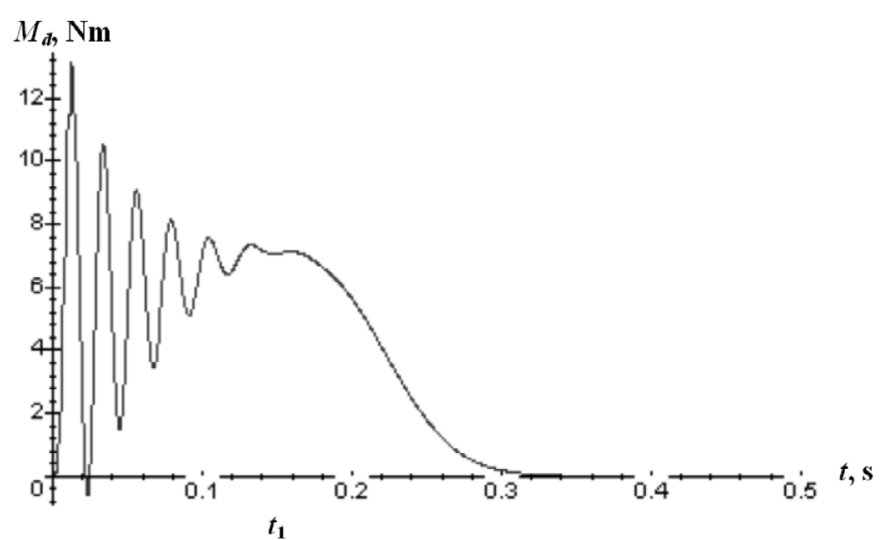

Fig. 1. Characteristics of the change of the dynamic moment of an electric motor.

obtain the following expressions for the frequency characteristics of the measurement means of the dynamic moment and the square of the modulus of the frequency characteristics, respectively:

$$
\begin{gathered}
S(j \omega)=\frac{K}{-\omega^{2}+j 2 \nu \omega_{p} \omega+\omega_{p}^{2}} ; \\
\text { see equation (18) above. }
\end{gathered}
$$

The input signal (on the right-hand side of differential Eq. (15)), on the basis of a priori information obtained during research on the characteristics of the change in the dynamic moment of the electric motor (Fig. 1), can be described by a mathematical model

$$
M_{\mathrm{c}}(t)=M_{k} e^{-\nu \omega_{0} t},
$$

where:

$M_{k}$ is the value of the torque at the point when power is cut to the stator of the electric motor;

$\omega_{0}$ is the cyclic frequency of the input signal.

The transfer function of the input signal (19) has the form

$$
X(p)=\frac{M_{k}}{p+\nu \omega_{0}} .
$$

Turning to the frequency domain, the spectral function of an input signal may be represented by:

$$
X(j \omega)=\frac{M_{k}}{j \omega+\nu \omega_{0}} .
$$

The square of the modulus of the spectral function of the input signal after separating the real and imaginary parts, and related mathematical transformation may be described by the expression

$$
|X(\mathrm{j} \omega)|^{2}=\frac{M_{k}^{2}\left(\omega^{2}+\nu^{2} \omega_{0}^{2}\right)}{\omega^{4}+2 \omega^{2} \nu^{2} \omega_{0}^{2}+\nu^{4} \omega_{0}^{4}} .
$$

Substituting the expressions of the square of the modulus of the spectral function of the input signal (22) and the square of the modulus of the frequency characteristics of the dynamic moment of the measurement means (18) in the formula for the evaluation of dynamic uncertainty (9), we obtain an expression which describes the dynamic uncertainty of the measurement means of the dynamic moment in the frequency domain from zero to infinity:

$$
\text { see equation (23) above. }
$$

In order to solve (23) with nominal values for the impact coefficients, and the construction characteristics of the changes in dynamic uncertainty we used the Maple mathematical package.

In as much as the method of measurement of the dynamic moment is down to powering off the stator of the electric motor, after the transition process and subsequent measurement of the dynamic moment in its self-braking mode, and the rotor speed varies from the nominal value of the frequency of the electricity grid at $50 \mathrm{~Hz}$ to that of when the rotor comes to a complete stop, then we shall research the characteristics of the changes to dynamic uncertainty at the start of the measurement (at the upper limit of measurement) of the dynamic moment at a rated frequency of $50 \mathrm{~Hz}$, and at the lower limits of measurement of the dynamic moment with decreasing frequency of the input signal to $1 \mathrm{~Hz}$, in the frequency domain from 0 to $20 \mathrm{kHz}$.

Substituting the nominal numerical values of the variables included in the equation (23) where $g=9.81 \mathrm{~m} / \mathrm{s}^{2}$, $K 1=489.89 \mathrm{~N}, J_{\mathrm{c}}=0.02 \mathrm{~N} \mathrm{~m}^{2}, M_{k}=8 \mathrm{~N} \mathrm{~m}$, $C=3000 \mathrm{~N} \mathrm{~m} / \mathrm{grad}, P=0.75 \mathrm{~N} \mathrm{~ms} / \mathrm{grad}$, we may obtain characteristics of the change in dynamic uncertainty of the measurement means, when measuring the dynamic moment in the range of 8 to $0.1 \mathrm{~N} \mathrm{~m}$ at a nominal rotor speed at $(50 \mathrm{~Hz})$ (Fig. 2), and the minimum frequency of the rotor speed $(1 \mathrm{~Hz})$ (Fig. 3), which are built into the environment of the Maple mathematical package. 


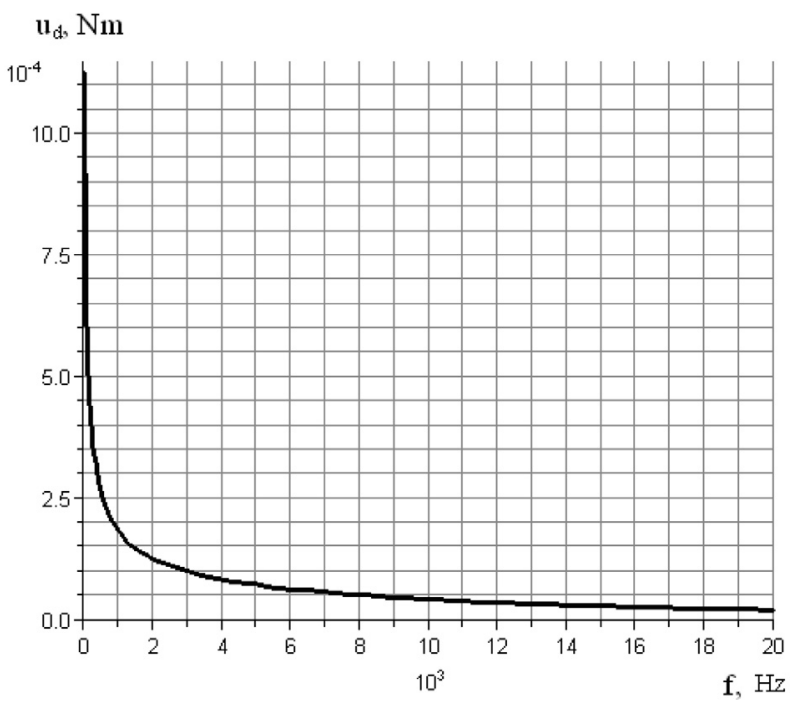

Fig. 2. Characteristics of the changes in the dynamic uncertainty of the dynamic moment of the measurement means at the start of measurement in the frequency range from 0 to $20 \mathrm{kHz}$.

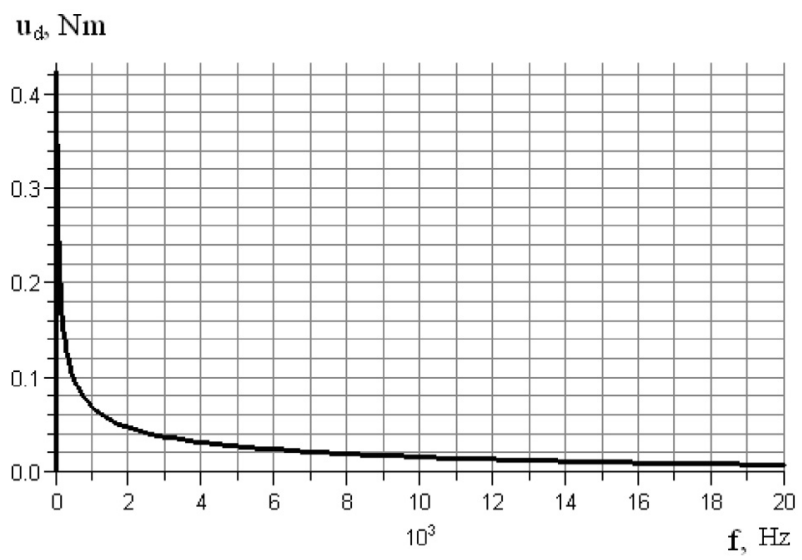

Fig. 3. Characteristics of the changes in the dynamic uncertainty of the dynamic moment of the measurement means at the end of measurement in the frequency range from 0 to $20 \mathrm{kHz}$.

As shown in Figures 2 and 3, the maximum values of the dynamic uncertainty of the dynamic moment of the measurement means in the range of measurement of the dynamic moment from 8 to $0.1 \mathrm{~N} \mathrm{~m}$ at the nominal frequency of a rotor speed of $50 \mathrm{~Hz}$, do not exceed $1.2 \times 10^{-3}$ (Fig. 2), and at the end of the measurement range when $u_{d}=0.43 \mathrm{~N} \mathrm{~m}$. Consequently, the standardised value of the dynamic uncertainty should equal a maximum (amplitude) value of the dynamic uncertainty, which appears at the lower limit of the measurement of the dynamic moment.

For convenience, we may perceive the obtained values of the dynamic uncertainty of the measurement means of the dynamic moment, we may calculate the relative

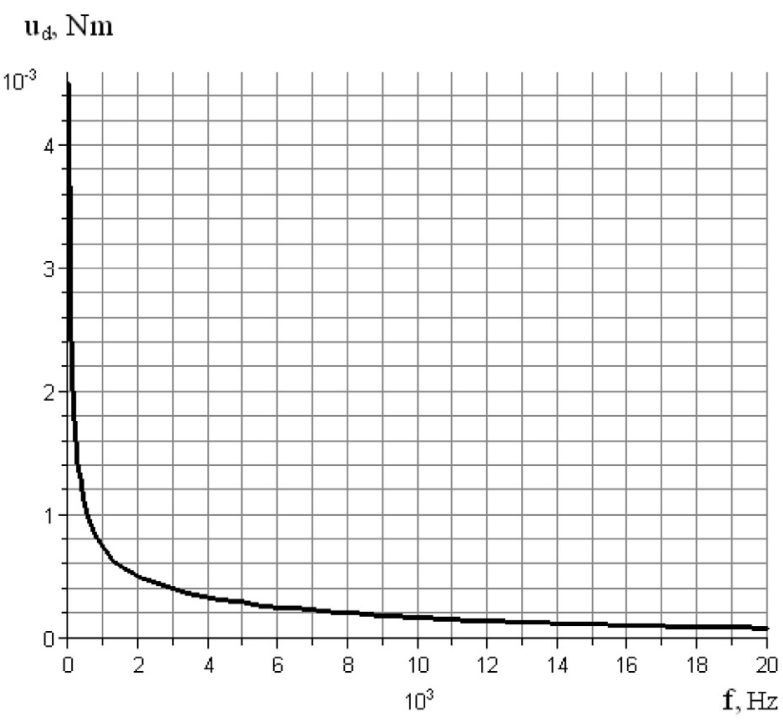

Fig. 4. Characteristics of change in the dynamic uncertainty of the measurement means dynamic moment at the start of the measurement range in the frequency domain to $20 \mathrm{kHz}$.

dynamic uncertainty of the measurement means, with formula (24)

$$
\tilde{u}_{d 1}=\frac{u_{d 1}}{M_{\max 1}} 100 \%=\frac{0.43}{8} 100 \%=5.38 \% .
$$

From the results of the evaluation of the dynamic uncertainty of the measurement means dynamic moment which was based on the frequency method of the evaluation of the dynamic uncertainty, we may see that the relative dynamic uncertainty is $5.38 \%$, where the range of measurement of the dynamic moment is 8 to $0.1 \mathrm{~N} \mathrm{~m}$.

In order to research the characteristics of the change in the dynamic uncertainty of the measurement means dynamic moment, when measuring the dynamic moment in the range of change from 30 to $0.1 \mathrm{~N} \mathrm{~m}\left(M_{k}=\right.$ $30 \mathrm{~N} \mathrm{~m}$ ), the characterization of dynamic uncertainty was constructed using the Maple mathematical package for the frequency domain up to $20 \mathrm{kHz}$ for the upper limit of the dynamic moment measurement (Fig. 4) and the lower limit of the measurement of the dynamic moment (Fig. 5). This was obtained on the basis of the metrological model of dynamic uncertainty of the dynamic moment measurement means (23).

As shown in Figures 4 and 5, the maximum value of the dynamic uncertainty of the dynamic moment measurement means in the range of change of the dynamic moment from 30 to $0.1 \mathrm{~N} \mathrm{~m}$ at the beginning of the measurement, and after the completion the transition period of the electric motor, is $4.5 \times 10^{-3} \mathrm{~N} \mathrm{~m}$ (Fig. 4), while at the end of the measurement the dynamic uncertainty of measurement means is $u_{d 2}=1.6 \mathrm{~N} \mathrm{~m}$. The standardised value of the dynamic uncertainty of the measurement means dynamic moment changes in the range of change from 30 to $0.1 \mathrm{~N} \mathrm{~m}$, the maximum (largest) value of dynamic uncertainty equates to $\left(u_{d 2}=1.6 \mathrm{~N} \mathrm{~m}\right)$, which is 


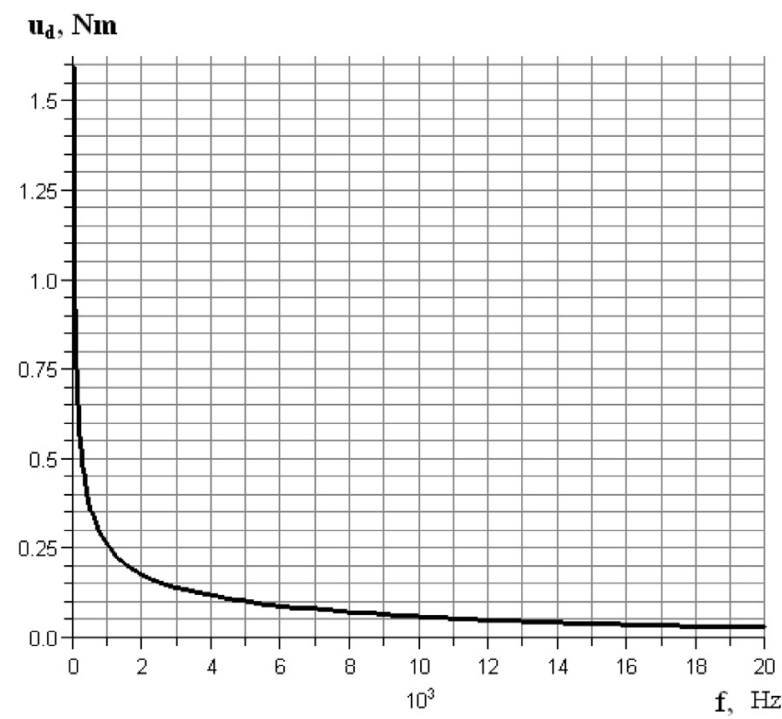

Fig. 5. Characteristics of change in the dynamic uncertainty of the measurement means dynamic moment at the end of the measurement range in the frequency domain to $20 \mathrm{kHz}$.

shown at the end of the measurement range (at the lower limit of measurement) of the dynamic moment.

In order to conveniently observe the obtained values of the dynamic uncertainty of the measurement means dynamic moment in the measurement range from 30 to $0.1 \mathrm{~N} \mathrm{~m}$, we may calculate the relative dynamic uncertainty of the measurement means by formula (24)

$$
\tilde{u}_{d 2}=\frac{u_{d 2}}{M_{\max 2}} 100 \%=\frac{1.6}{30} 100 \%=5.33 \% .
$$

From the results of the evaluation of the dynamic uncertainty of the measurement means dynamic moment of the $\mathrm{EM}$ in the range from 30 to $0.1 \mathrm{~N} \mathrm{~m}$, it may be seen that that the relative dynamic uncertainty of the measurement means is $5.33 \%$.

Thus, the values obtained of the dynamic uncertainty of the measurement means must be added to the values of the combined uncertainty of the measurement results, which include residual systematic effects, which may be observed, because of the limited properties of the constituent elements of the measurement means (in terms of precision). These may be measured by type B and experimental uncertainty of measurement of type A by the formula

$$
u_{c d}=\sqrt{u_{c}^{2}+u_{d}^{2}} .
$$

When substituting the previously obtained values for the standard uncertainties of type B and type A (a priori and a posteriori, or just a priori), which together constitute $u_{c}=88.99 \times 10^{-3} \mathrm{~N} \mathrm{~m}$, and the dynamic uncertainty of the measurement means dynamic moment that is $u_{d 1}=0.43 \mathrm{~N} \mathrm{~m}$ in the range of measurement from 8 to $0.1 \mathrm{~N} \mathrm{~m}$ in equation (25), we obtain the value of the combined uncertainty of the measurement means dynamic moment as $u_{\mathrm{cd} 1}=0.45 \mathrm{~N} \mathrm{~m}$.

When substituting the values for the standard uncertainties of the measurement means dynamic moment $u_{d 2}=1.6 \mathrm{~N} \mathrm{~m}$, in the range of measurement from 30 to $0.1 \mathrm{~N} \mathrm{~m}$ together with the combined $u_{c}=88.99 \times 10^{-3} \mathrm{~N} \mathrm{~m}$ which was evaluated for statistical indicators in equation (25), we obtain the value of the combined uncertainty of the results of the measurement of the dynamic moment as $u_{c d 2}=1.61 \mathrm{~N} \mathrm{~m}$.

Enhanced uncertainty of the measurement of the dynamic moment for the measurement range from 8 to $0.1 \mathrm{~N} \mathrm{~m}$ and from 30 to $0.1 \mathrm{~N} \mathrm{~m}$, may be calculated by the formulae:

$U_{d 1}=k_{\mathrm{p}} u_{c d 1}=1.96 \times 0.45=0.88 \mathrm{~N} \mathrm{~m}$, where $P=0.95 ;$

$U_{d 2}=k_{\mathrm{p}} u_{c d 2}=1.96 \times 1.61=3.16 \mathrm{~N} \mathrm{~m}$, where $P=0.95$.

In order to conveniently observe the obtained values of uncertainties of the dynamic moment in the measurement range from 30 to $0.1 \mathrm{~N} \mathrm{~m}$, we may calculate the relative combined uncertainties of the measurement results of the dynamic moment, which equal $5.63 \%$ in the measurement range of the dynamic moment from 8 to $0.1 \mathrm{~N} \mathrm{~m}$ and $5.37 \%$ in the measurement range of the dynamic moment from 30 to $0.1 \mathrm{~N} \mathrm{~m}$ by means of formula (24).

\section{Conclusion}

Thus, the frequency method to evaluate the dynamic uncertainty of measurement means, and the metrology model of dynamic uncertainty of the measurement means dynamic moment, has been developed to allow us to determine the characteristics of precision of measurement means that operate in dynamic modes based on information obtained on frequency characteristics of the measurement means, and the spectral function of the input signal model. This permits us to research the behaviour of their changes and determine the amplitude values of dynamic uncertainty of measurement means over a wide frequency domain.

This method ensures the uniformity of dynamic measurements and allows us to present and compare the dynamic uncertainty of measurement means used in dynamic measurements, with results obtained by other laboratories and research centres of leading nations around the world.

The proposed approach to the evaluation of dynamic uncertainty of measurement means can be used for any measurement means characterized by dynamic components of any type.

\section{References}

1. ISO/IEC Guide 98-1:2009, Uncertainty of measurementPart 1: Introduction to the expression of uncertainty in measurement (Geneva, Switzerland, ISO, 2009), p. 32.

2. IEC GUIDE 115-2007, Application of uncertainty of measurement to conformity assessment activities in the electrotechnical sector (Geneva, Switzerland, IEC, 2007), 54 p. 
3. Evaluation of measurement data. Guide to the expression of uncertainty in measurement, JCGM 100, GUM 1995 with minor corrections, 1st edn. (2008).

4. C. Elster, S. Eichstädt, A. Link, Uncertainty evaluation of dynamic measurements in line with GUM, In XIX IMEKO World Congress on Fundamental and Applied Metrology (2009)

5. O.M. Vasilevskyi, Calibration method to assess the accuracy of measurement devices using the theory of uncertainty, Int. J. Metrol. Qual Eng. 5, 403 (2014)

6. T.J. Esward, C. Elster, J.P. Hessling, Analysis of dynamic measurements: new challenges require new solutions, in Proc. of XIX IMEKO World Congress on Fundamental and Applied Metrology (2009)

7. S. Eichstädt, A. Link, C. Elster, Dynamic Uncertainty for Compensated Second-Order Systems, Sensors, 10, 7621 (2010)
8. C. Elster, A. Link, Analysis of dynamic measurements: compensation of dynamic error and evaluation of uncertainty, In Advanced Mathematical \& Computational Tools in Metrology VIII, Series on Advances in Mathematics for Applied Sciences (World Scientific New Jersey, 2009), Vol. 78, pp. 80-89

9. O.M. Vasilevskyi, Advanced mathematical model of measuring the starting torque motors, Tekhnichna elektrodinamika, 6, 76 (2013)

10. S. Eichstädt, Analysis of Dynamic Measurements Evaluation of dynamic measurement uncertainty (2012)

11. O. Vasilevskyi, V. Kucheruk, I. Kurytnik, An approach to the evaluation of dynamic uncertainty in measurement using non-statistical techniques (Pomiary, Automatyka, Kontrola, 2014), Vol. 60, 11, pp. 997-1001

12. O.M. Vasilevskyi, V. Yu. Kucheruk, Fundamentals of the theory of measurement uncertainty (2013), p. 224 